\title{
Penicillium commune affects textural properties and water distribution of hard and extra-hard cheeses
}

\author{
Miguel Jurado and Carlos Javier Vicente
}

\begin{abstract}
We analyzed the effects of growth of Penicillium commune, one of the most frequent fungal species associated with cheese, on the water diffusion and texture of hard and extra-hard cheeses. A total of 36 hard cheese blocks and 36 extra-hard cheese blocks were manufactured, salted at different levels $(0.5,1.25$ and $2 \% \mathrm{w} / \mathrm{w})$, and assigned to different treatments (control and inoculated). Cheese texture was analyzed using a penetration needle probe at 2 and 5 weeks after ripening. Firmness, defined as the maximum force detected in the penetration probe, was higher in both hard and extra-hard inoculated cheese blocks compared with the control. In addition, the presence of fungal growth on cheese rind increased the total work of penetration (a measure of resistance to probe penetration), but only in extra-hard cheeses, suggesting that the moisture of cheese might be affecting the growth capacities and perform-ance of $P$. commune. The change in textural properties of cheeses was linked to desiccation of the upper $0.5-\mathrm{cm}$ rind layer mediated by $P$. commune.

Cheese rinds are frequently colonized by filamentous fungi during the ripening and storage periods. In some cheese varieties, certain fungal species are intentionally added as starter cul-tures. For example, Penicillium camemberti is a fungal species whose mycelium gives rise to the so-called 'bloomy rind cheeses' (e.g. Camembert, Brie) imparting a whitish velvety aspect. In other cheese varieties, such as Saint Nectaire or Tomme, fungal starters might not be added, but naturally-occurring fungi are encouraged to grow, typically by ripening cheese wheels in high-humidity environments, such as caves or chamber rooms with adjusted conditions. The rinds of these cheeses host diverse microbial communities domi-nated by filamentous fungi, and less abundant yeasts and bacteria (Wolfe et al., 2014). These complex microbiomes form very well-defined biofilms or 'natural rinds' on the cheese surface.

Besides the situations mentioned above, fungi can also be spoilage agents in many cheese varieties. Strategies such as vacuum packaging or application of coatings with fungistatic activity can be implemented to prevent fungal contamination (Nardoni et al., 2018). However, fungal growth is almost unavoidable, especially when cheeses are ripened for long periods, so it is necessary to brush the mold off the cheese wheels periodically (Marín et al., 2018).

Irrespective of the cause of fungal presence in cheese, it is important to consider that molds not only modify the rind characteristics, but also have the ability to alter the organoleptic char-acteristics of cheese (Ceugniez et al., 2017). These changes are considered positive or negative depending on the fungal species involved and the variety of cheese considered. One of the organoleptic attributes susceptible to modification by fungi is texture, which is considered a key property of cheese. Texture is a criterion of cheese hardness, which is broadly defined as soft ( $>55 \%$ moisture; e.g. Cottage), semi-soft (41-55\% moisture; e.g. Muenster), hard (35-40\% moisture; e.g. Cheddar) or extra-hard (<35\% moisture; e.g. Parmesan), although these labels and the category boundaries may vary depending on the numerous existing clas-sification systems (McSweeney et al., 2004). The texture is influenced not only by moisture content of the cheese (as water content decreases, firmness increases), but also by the integrity of the microstructure of protein matrix (Sousa et al., 2001).

Proteolysis in cheese is mostly caused by the residual coagulant, indigenous milk enzymes and bacterial starters (Sousa $e t$ al., 2001). Many proteases secreted by fungal species commonly used in cheese production have been identified and characterized (McSweeney et al., 2004; Button and Dutton, 2012; Sacristán et al., 2012). Some fungal proteases are even asserted to have a specific role in the decrease of cheese firmness (Boutrou et al., 2006; Fernández-Bodega et al., 2009; Jurado and Ruiz-Navarro, 2018). However, the focus has been on soft or semi-soft cheeses and, for several reasons, it is difficult to extrapolate the data to hard or extra-hard cheeses. On the one hand, the intrinsic chemical and structural
\end{abstract}


characteristics of softer cheeses, mostly related to their higher water contents, are quite different to the harder varieties and, on the other hand, the fungal species associated with cheeses with different moisture contents might differ considerably because the water activity critically affects the distribution and dynamics of fungal populations (Marín et al., 2015).

Therefore, this study investigated the effects of fungal growth on the textural properties of hard and extra-hard cheeses. Penicillium commune was specifically selected as it is one of the most frequently occurring spoilage fungi on cheese (Lund et al., 2003), and it is predominantly present on cheeses with low water contents (Hocking and Faedo, 1992; Lund et al., 1995; Marín et al., 2015). Different salt concentrations and ripening times were considered to obtain a variety of conditions, and the results were compared with the control treatments.

\section{Material and methods}

\section{Cheese manufacture}

Hard and extra-hard cheeses were prepared from the same batch of cow milk, which was obtained from a local farm in Guadalix de la Sierra (Madrid, Spain). Milk was pasteurized at $75^{\circ} \mathrm{C}$ for $20 \mathrm{~s}$ in a plate heat-exchanger and cooled at $20^{\circ} \mathrm{C}$ using the regeneration section of the pasteurizer. Milk was then transferred to a vat, heated to $32^{\circ} \mathrm{C}$ and a mesophilic starter culture was added (Choozit MA 4001 LYO; Danisco, Copenhagen, Denmark). Milk was ripened for $30 \mathrm{~min}$, and $0.075 \% \mathrm{CaCl}_{2}$ and $0.02 \%$ chymosin (Laboratorios Arroyo, Santander, Spain) were added to the vat. After $35 \mathrm{~min}$, the curd was cut into cubes of approximately 1 $\mathrm{cm}^{2}$ and the temperature was raised to $37^{\circ} \mathrm{C}$ for hard cheeses and $42^{\circ} \mathrm{C}$ for extra-hard cheeses. Curd cubes were stirred for 60 (hard cheeses) or $90 \mathrm{~min}$ (extra-hard cheeses), before draining the whey. Curd was then placed into molds, and hard cheeses were pressed in two steps ( $0.1 \mathrm{MPa}, 30 \mathrm{~min} ; 0.2 \mathrm{MPa}, 90 \mathrm{~min})$, whereas extrahard cheeses were pressed in three steps $(0.1 \mathrm{MPa}, 30 \mathrm{~min} ; 0.2$ $\mathrm{MPa}, 30 \mathrm{~min}$; and $0.3 \mathrm{MPa}, 60 \mathrm{~min}$ ).

After press, cheeses were surface-salted to three different final $\mathrm{NaCl}$ concentrations $(0.5,1.25$ or $2 \% \mathrm{w} / \mathrm{w})$, and transferred to a drying chamber maintained at $12 \pm 1^{\circ} \mathrm{C}$ and $70 \%$ relative humidity $(\mathrm{RH})$ for $24 \mathrm{~h}$ to remove the excess water from within the cheese surface. From all the cheeses elaborated, 36 hard cheeses and 36 extra-hard cheeses were selected to minimize the cheese size variation within each group. The selected cheeses were 15 $\mathrm{cm}$ in diameter, with weight and height dimensions of $353 \pm 26$ $\mathrm{g}$ and $5.5 \pm 0.3 \mathrm{~cm}$ (hard cheeses) and $319 \pm 21 \mathrm{~g}$ and $5.2 \pm 0.2$ $\mathrm{cm}$ (extra-hard cheeses). The final $\mathrm{pH}$ of the cheeses ranged from 5.3 to 5.4 .

\section{Cheese inoculation and ripening}

Cheeses (triplicate) with different contents of salt were assigned to two different treatments (control and inoculated) and ripening periods ( 2 and 5 weeks). Control cheeses were coated by immersion with a solution of polyvinyl acetate plastic (Manuel Riesgo, Madrid, Spain) at 20\% supplemented with $0.1 \%$ natamycin (Danisco, Madrid, Spain) and $4 \%$ potassium sorbate (Sigma, Madrid, Spain) as antifungal compounds to avoid any fungal contamination. Inoculated cheeses were also coated with a solution of polyvinyl acetate at $20 \%$ containing a spore suspension of $P$. commune PCOM5 (Marín et al., 2015) to a final concentration of $6 \times$ $10^{5}$ spores $/ \mathrm{ml}$. Spores had been previously collected from 10-d-old cultures grown on Sabouraud dextrose agar (Oxoid, Madrid, Spain). After application of the surface treatment, control and inoculated cheeses were transferred to a ripening chamber maintained at $12 \pm 1^{\circ} \mathrm{C}$ and $85 \% \mathrm{RH}$ for either 2 or 5 weeks. The ripening chambers had been thoroughly cleaned and sanitized before the study started. Cheeses were inspected daily to visualize fungal growth and turned over every 1 or $2 \mathrm{~d}$ throughout the ripening period.

\section{Penetrometer assays}

Mechanical strength tests were performed using a Stevens LFRA texture analyzer (Brookfield Engineering Laboratories, Middleboro, MA, USA). A needle probe was used to penetrate the cheeses ripened for 2 and 5 weeks of incubation (control and inoculated) at a constant speed of $0.5 \mathrm{~mm} / \mathrm{s}$ to a depth of $13.5 \mathrm{~mm}$ beneath the suspension surface, resulting in a sampling time of $27 \mathrm{~s}$. Cheeses were equilibrated at room temperature $\left(20^{\circ} \mathrm{C}\right)$ prior to testing, and every cheese was measured at different and equidistant points (three per side of the cheese).

The mass component of force $(\mathrm{g})$ was recorded, and the respective force-distance curves were generated using Excel (Microsoft). Firmness was defined as the maximum force detected during the penetration assay, and the total work of penetration was converted to single values of mechanical strength by calculating the area under the force-displacement curve (AUC) with trapezoidal integration technique using Excel.

\section{Determination of weight loss and water contents of cheese}

Weight loss of cheese was measured by monitoring the weight changes every $7 \mathrm{~d}$ on the batch of cheeses ripened for 5 weeks, and calculated as a percentage of weight loss from the initial cheese weight.

At the end of the ripening period ( 2 or 5 weeks), cheeses were cut along the vertical axis to obtain different portions: (i) rind, defined as the first $0.5 \mathrm{~cm}$ of the surface of cheese, (ii) sub-rind, defined as the portion located between 0.5 and $1 \mathrm{~cm}$, and (iii) core, corresponding to the rest of the cheese. Their respective water contents were determined by measuring the weight loss at $105^{\circ} \mathrm{C}$ for $24 \mathrm{~h}$ until constant weight.

\section{Statistical analysis}

All data are expressed as mean \pm (sD) (triplicate). Differences between the inoculated and control cheeses were tested by oneway ANOVA using Statgraphics Centurion XVI (Statistical Graphics Corp., Herndon, VA, USA). Post hoc comparisons were achieved using Tukey's HSD test. Statistical significance was set at $P<0.05$.

\section{Results}

\section{Cheese inspection}

All cheeses that were inoculated showed fungal growth after 6-7 $\mathrm{d}$ and were completely covered by mycelium after 10-12 $\mathrm{d}$. No evident differences in the progression of growth were found among inoculated cheeses containing different percentages of salt, or between hard and extra-hard cheeses. Control cheeses remained with no visible fungal growth throughout the ripening period. 


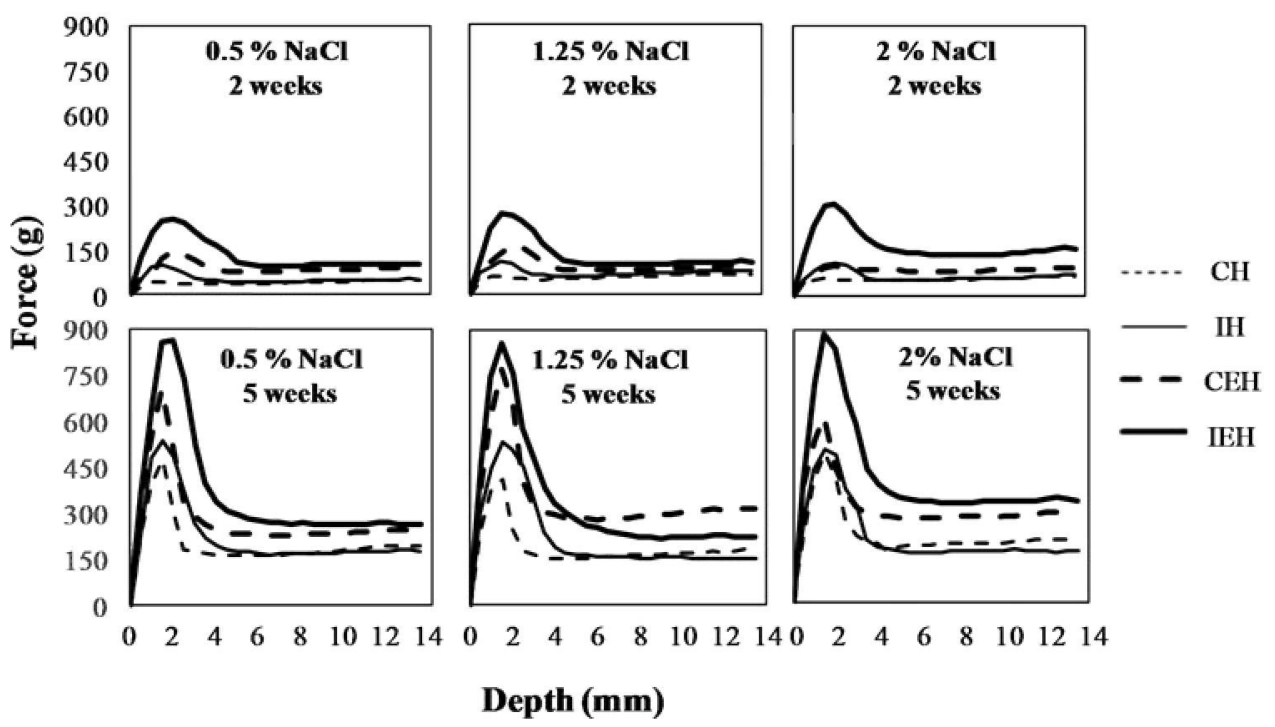

Fig. 1. Force (g) evolution during penetration assays of control (C) and inoculated (I) hard (H) and extra-hard (EH) cheeses ripened for 2 or 5 weeks and salted with different percentages of $\mathrm{NaCl}\langle 0.5,1.25$ and $2 \% \mathrm{w} / \mathrm{w})$. Data represent the mean of three replicates.

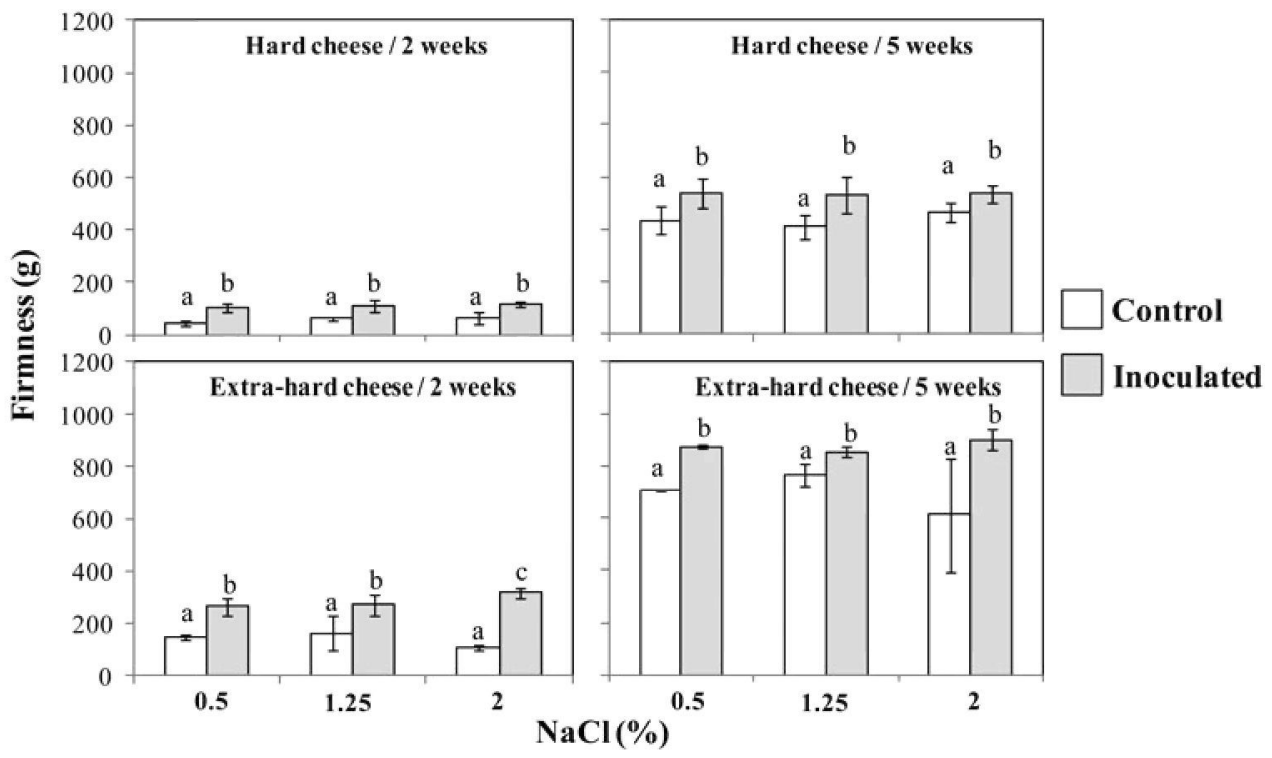

Fig. 2. Firmness (F) or maximum force achieved during penetration of hard and extra-hard cheeses with different contents of salt $(0.5,1.25$ and $2 \%$ w/ $W$ ) and ripened for 2 or 5 weeks. Samples not significantly different share the same letter. Error bars denote the SE of the mean of three replicates.

\section{Penetration assays}

Force-displacement curves of the penetration probes corresponding to hard and extra-hard cheeses with different salt concentrations are shown in Figure 1. Most of the curves displayed a convex-like shape, characterized by a maximum peak after the first $12 \mathrm{~mm}$ of depth (coinciding with the maximum force of the rind until its breakage), followed by a decrease in resistance to penetration and a final plateau. The exceptions were the curves corresponding to non-inoculated hard cheeses ripened for 2 weeks, which were more or less flat, thereby indicating the absence of a consolidated rind. The convex-like part of the curve was for inoculated cheeses compared with control cheeses, and consequently, the beginning of the plateau was right-shifted to a higher depth of needle penetration.
Figure 2 illustrates the cheese firmness (defined as the maximum force detected during the penetration assays). This parameter increased with the duration of the ripening period and was higher in extra-hard cheeses than in hard cheeses, and in inoculated that non-inoculated cheeses. Salt concentration did not influence cheese firmness, except for inoculated extra-hard cheeses salted at $2 \%$ and ripened for 2 weeks, which showed higher resistance to penetration in comparison to their counterparts salted at 0.5 and $1.25 \%$. However, these differences in firmness disappeared after 5 weeks of ripening.

Figure 3 display the total work of penetration (AUC), which is a measure of cheese strength and resistance to probe penetration. AUC increased with the ripening time and was higher in extrahard cheeses, but there were no differences between the control 


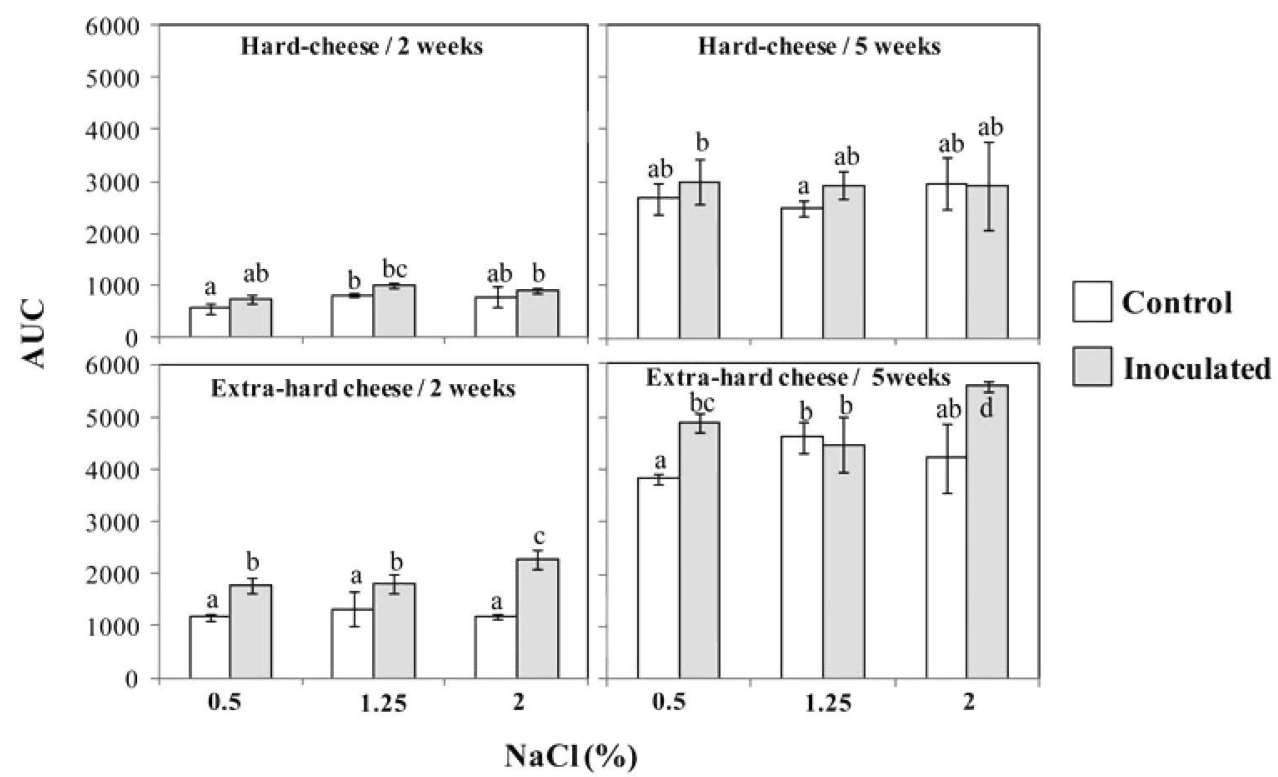

Fig. 3. Total work of penetration expressed as the area under curve (AUC) of hard and extra-hard cheeses with different contents of salt ( $0.5,1.25$ and $2 \%$ w/ $w$ ) and ripened for 2 or 5 weeks. Samples not significantly different share the same letter. Error bars denote the SE of the mean of three replicates.
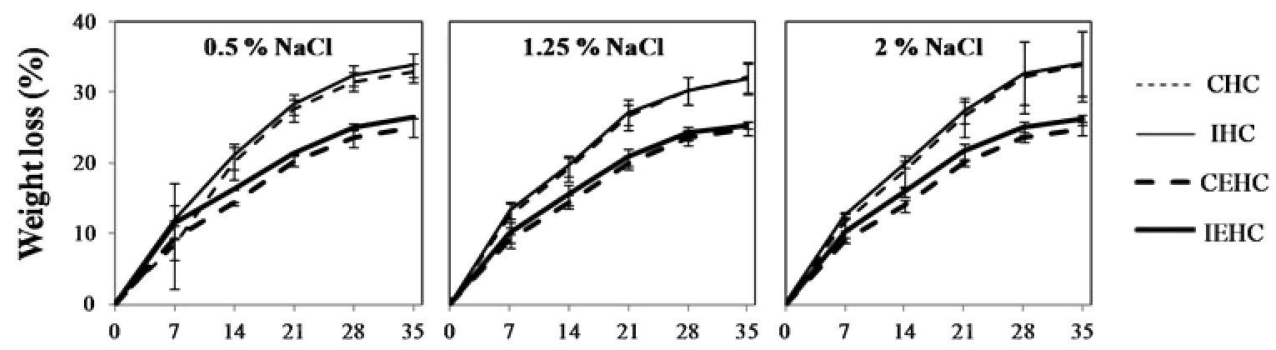

Time(days)

Fig. 4. Percentage of cheese weight loss evolution of hard and extra-hard cheeses with different contents of salt $(0.5,1.25$ and $2 \%$ w/w) and ripened for 2 or 5 weeks. Error bars denote the SE of the mean of three replicates.

and inoculated hard cheeses. However, among the extra-hard cheeses, AUC values were higher in the inoculated samples, except for those salted at $1.25 \%$ and ripened for 5 weeks, which were not significantly different. Salt concentration did not affect effect AUC in hard cheeses, but salting at $2 \%$ increased the work of penetration in extra-hard cheeses ripened for 2 or 5 weeks.

\section{Weight loss and water contents of cheese}

Figure 4 reveals the weekly weight loss of cheeses ripened for 5 weeks. Mass losses were higher at the beginning of the ripening period in both hard and extra-hard cheeses. No statistical differences were found between the control and inoculated cheeses or among cheeses with different percentages of salt, but mass losses were higher in hard cheeses through the ripening period.

The final water contents were determined in different portions of the cheese after 5 weeks of ripening (Fig. 5). Rind and subrinds presented lower water contents than cheese cores. No differences in water contents were detected between the cores of control and inoculated cheeses. On the contrary, the rinds of cheeses inoculated with $P$. commune presented lower water contents than rinds obtained from the control cheeses, regardless of salt concentration. Sub-rinds of inoculated hard cheeses salted with 0.5 and $2 \%$ also presented lower water contents than the controls.

\section{Discussion}

The ripening period of cheeses is marked by progressive evaporation of water from the cheese surface and a subsequent rind consolidation. Depending on the previous technological procedures performed during the successive cheese-making steps, as well as the environmental conditions present in the ripening rooms, the chemical and microstructural characteristics of these rinds will greatly diverge among different cheese varieties. Several studies have shown that substrate has an important, sometimes limiting, role in the distribution and growth of fungal species in cheese (Marín et al., 2014, 2015). The results obtained in this work showed a rapid progression of growth of $P$. commune on both hard and extra-hard cheeses at all salt concentrations that would support the great ubiquity of $P$. commune that has been described in the literature among different varieties of cheese (Lund et al., 2003). 


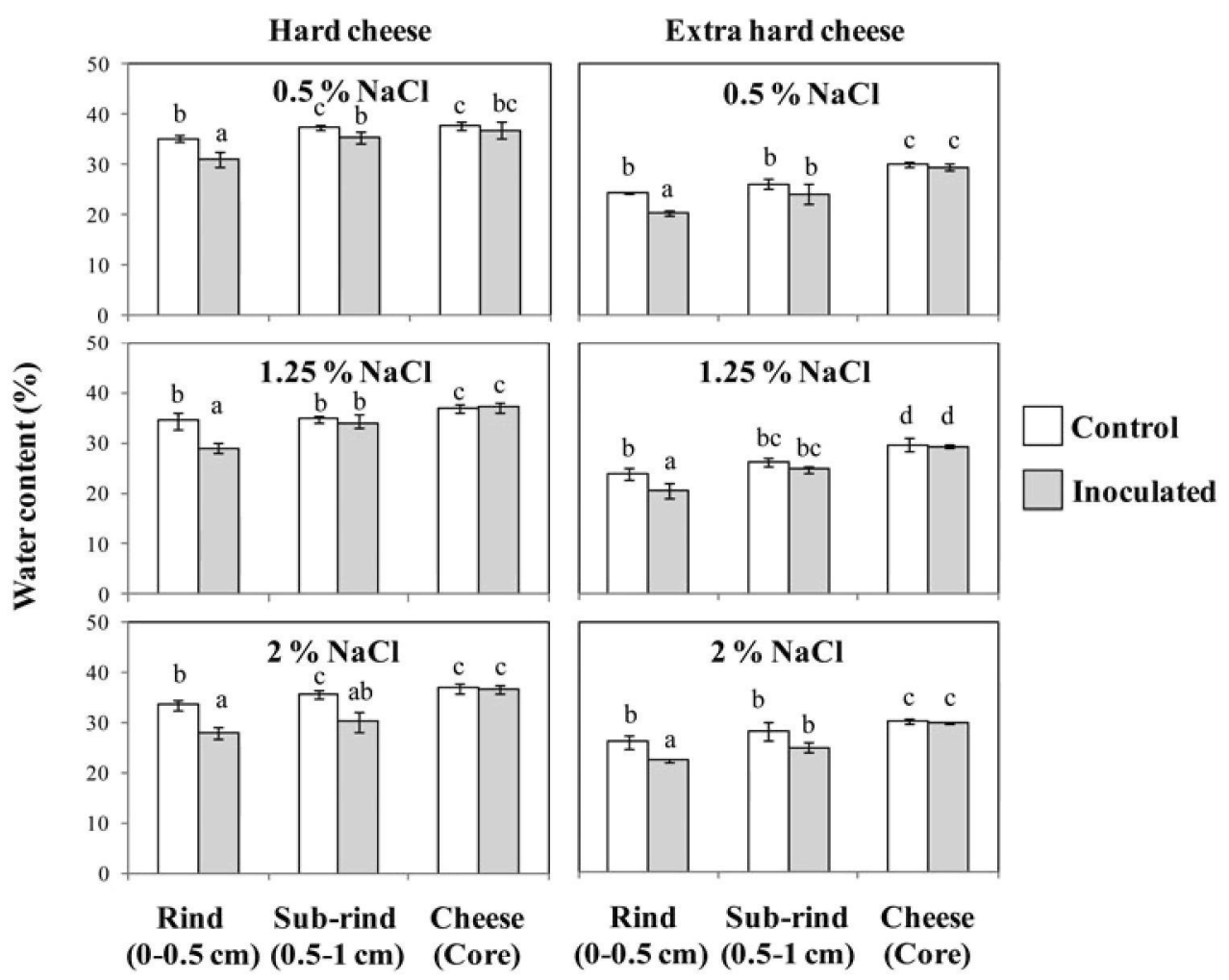

Fig. 5. Percentage of water content in different parts (rind, sub-rind and whole cheese) of hard and extra-hard cheeses with different contents of salt (0.5, 1.25 and $2 \% \mathrm{w} / \mathrm{w}$ ) and ripened for 5 weeks. Samples not significantly different share the same letter. Error bars denote the $5 \mathrm{E}$ of the mean of three replicates.

The modification of the shapes of the penetration curves in both inoculated hard and extra-hard cheeses, characterized by a wider initial force peak might be due, at least in part, to the mere accumulation of mycelium biomass that would contribute to the formation of a thicker rind. In addition, the increase in the maximum force detected among the inoculated cheeses would also indicate a hardening effect on the rind due to $P$. commune. The increase in AUC detected among the inoculated extrahard cheeses could suggest that a lower moisture content could enhance the growth of $P$. commune, and thereby, its ability to modify the textural properties of cheese. Nevertheless, it is important to note that resistance on penetration remained higher on inoculated extra-hard cheeses than in controls even after breakage of the rind, as evidenced by the higher plateaus reached after the initial peak. Therefore, our results indicate that fungal growth modified not only the texture of the rind but also the texture under the rind. This scenario could have an important impact on the organoleptic properties of these cheeses, because a more heterogeneous texture is generally considered to be less appealing to consumers. The inconvenience of these changes, however, should be carefully evaluated in different cases. For example, a firmer rind could also represent an advantage against mechanical damages of cheese. Some producers of Manchego, the most important pressed cheese produced in Spain, intentionally allow the growth of adventitious fungi during the first weeks of ripening, assuming that it improves the flavor and the consolidation of the rind. After a few weeks, the mold is usually scrapped once the rind is firm, and an antifungal coating is further applied to avoid fungal growth during the rest of the aging period. This potentially beneficial consequence of fungal growth merits thorough analysis not only in Manchego cheese but also in the production of other hard and extra-hard varieties, especially considering the ubiquity of $P$. commune.

Interestingly, regarding firmness, our results contrast with the reports on soft cheeses, which found that firmness decreased in soft cheeses as a result of the proteolytic activity of fungi (Boutrou et al., 2006; Fernández-Bodega et al., 2009; Jurado and Ruiz-Navarro, 2018). Although P. commune stands out as a proteolytic microorganism (Papagianni, 2014; Alapont et al., 2015), several factors could contribute to explain the results found in our study. On the one hand, in comparison to soft cheeses, the lower water content and water activity of harder cheeses could reduce the performance of proteases, considering that both parameters influence the enzymatic activity during the ripening process (Saurel et al., 2004). This modification in protease activity could help to explain the role of fungi on proteolysis and firmness reduction described in cheeses with comparatively high water contents, such as Camembert (Leclercq-Perlat et al., 2004) and smear cheeses (Bonaiti et al., 2004). On the other hand, it could be hypothesized that the distinctive rigid structure of hard and extra-hard cheeses could hamper the migration of fungal proteases from the rind to the core of the cheese. This effect will be expected to increase throughout the ripening period because, as our results show, water loss progresses during the maturation of cheese. However, even assuming that migration of $P$. commune proteases could be hindered, this could not completely explain why proteases were unable to reduce the firmness of the rind. In our experience in the cheese industry, hard and extra-hard cheeses that are surface-contaminated with fungi are occasionally prone to suffer proteolysis in the first few millimeters of the rind, revealed as a reduction in firmness and sometimes in severe loss of structure. It could be that only certain species and, or either, 
very long periods of ripening could eventually lead to intense proteolysis in hard or extra-hard cheeses.

The analysis of the distribution of water in different portions of cheese (rind, sub-rind and core) was of relevance because firmness is not only related to the integrity of the microstructure of the protein matrix but also to the moisture content. Growth of P. commune induced, in all cases, desiccation of the rind, which, in some cases also affected the sub-rind, but not the cheese cores. This loss of water would be the main factor explaining the changes in firmness and AUC. Several studies have attempted to model the water diffusion in cheese, which found that water mobility differ depending on the variety of cheese considered (Pajonk et al., 2003; Bonaïti et al., 2004; Saurel et al., 2004; Hélias et al., 2007). Gaucel et al. (2012), who proposed a generalized model for determining cheese mass loss during ripening based on the respiration of the microbiome and water evaporation, found that the respiratory activity of the microorganisms at the surface of ripening cheese was the most important factor to predict water diffusion. Concurrently, a metagenomic study performed with different types of rinds established that moisture was the factor that best correlated with microbial diversity (Wolfe et al., 2014). The authors further noted that Penicillium spp. was the dominant filamentous fungal genus detected in rinds with low water contents. Our results indicated that the presence of $P$. commune was able per se to modify the water fluxes in cheese, by increasing the water diffusion to the surface. These results reveal that the composition of the microbiome growing on the rind is not just a result of the intrinsic characteristics of the cheese matrix or the environmental factors present in the drying chambers, as has been stated until now. On the contrary, the microbiota seems to exert a significant and reciprocal influence on the resulting characteristics of the rind (and sub-rind), in this case on the moisture content.

Regarding the mechanisms of rind desiccation, a most plausible hypothesis might be that fungal hyphae of $P$. commune could increase the total surface of the cheese so that more water molecules could evaporate. However, the great microbial diversity present in cheese makes it necessary to direct future research toward providing evidence of the mechanisms involved in water transfer through the rind in the presence of different microbial consortia. This information would be beneficial to elucidate the specific role of these microorganisms in rind formation, and its influence on the textural properties of different cheese varieties.

In conclusion, inoculation of hard and extra-hard cheeses with Penicillium commune increased firmness in both types and increased the total work of penetration but only in extra-hard cheeses, suggesting that the moisture of cheese might be affecting the growth capacities and performance of $P$. commune. The change in textural properties of cheeses was linked to desiccation of the upper $0.5-\mathrm{cm}$ rind layer mediated by $P$. commune.

\section{References}

Alapont C, Martínez-Culebras PV and López-Mendoza MC (2015) Determination of lipolytic and proteolytic activities of mycoflora isolated from dry-cured teruel ham. Journal of Food Science and Technology 52, $5250-5256$.

Bonaiti C, Leclercq-Perlat MN, Latrille E and Corrieu G (2004) Deacidification by Debaryomyces hansenii of smear soft cheeses ripened under controlled conditions: relative humidity and temperature influences. Journal of Dairy Science 87, 3976-3988.

Boutrou R, Aziza M and Amrane A (2006) Enhanced proteolytic activities of Geotrichum candidum and Penicillum camemberti in mixed culture. Enzyme and Microbial Technology 39, 325-333.
Button JE and Dutton RJ (2012) Cheese microbes. Current Biology 22, 87-89. Ceugniez A, Taminiau B, Coucheney F, Jacques P, Delcenserie V, Daube G and Drider D (2017) Fungal diversity of 'Tomme d'Orchies' cheese during the ripening process as revealed by a metagenomic study. International Journal of Food Microbiology 258, 89-93.

Fernández-Bodega MA, Mauriz E, Gómez A and Martin JF (2009) Proteolytic activity, mycotoxins and andrastin $\mathrm{A}$ in Penicillium roquefort strains isolated from Cabrales, Valdeón and Bejes-Tresviso local varieties of blue-veined cheeses. International Journal of Food Microbiology 136, $18-25$.

Gaucel S, Guillermin H and Corrieu G (2012) A generalised model for cheese mass loss determination during ripening. Joumal of Food Engineering 110 , $109-116$.

Hélias A, Mirade PS and Corrieu G (2007) Modeling of camembert-type cheese mass loss in a ripening chamber: main biological and physical phenomena. Journal of Dairy Science 90, 5324-5333.

Hocking AD and Faedo M (1992) Fungi causing thread mould spoilage of vacuum packaged Cheddar cheese during maturation. International Journal of Food Microbiology 16, 123-130.

Jurado $\mathbf{M}$ and Ruiz-Navarro $\mathbf{P}$ (2018) Effects of fungal growth on the firmness of a cheese analogue formulated with different casein-to-fat ratios. LWT Food Science and Technology 90, 145-151.

Leclercq-Perlat MN, Buono F, Lambert D, Latrille E, Spinnler HE and Corrieu G (2004) Controlled production of camembert-type cheeses. Part I: microbiological and physicochemical evolutions. Journal of Dairy Research 71, 346-354.

Lund F, Filtenborg O and Frisvad C (1995) Associated mycoflora of cheese. Food Microbiology 12, 173-180.

Lund F, Nielsen AB and Skouboe P (2003) Distribution of Penicillium commune isolates in cheese dairies mapped using secondary metabolite profiles, morphotypes, RAPD and AFLP fingerprinting. Food Microbiology 20, 725-730.

Marín P, Palmero D and Jurado M (2014) Effect of solute and matric potential on growth rate of fungal species isolated from cheese. International Dairy Journal 36, 89-94.

Marín P, Palmero D and Jurado $\mathbf{M}$ (2015) Occurrence of moulds associated with ovine raw milk and cheeses of the Spanish region of Castilla La Mancha. International Journal of Dairy Technology 68, 565-572.

Marín P, Ginés C, Kochaki P and Jurado $\mathbf{M}$ (2018) Effects of water activity on the performance of potassium sorbate and natamycin as preservatives against cheese spoilage moulds. Irish Journal of Agricultural and Food Research 56, 85-92.

McSweeney PLH, Ottogalli G and Fox PF (2004) Cheese: an overview. In Fox PF, McSweeney PLH, Cogan TM and Guinee TP (eds), Cheese Chemistry, Physics and Microbiology Volume 2, 3rd Edn. London, UK: Elsevier Academic Press, pp. 1-23

Nardoni S, D'Ascenzi C, Caracciolo I, Mannaioni G, Papini RA, Pistelli L, Basma N and Mancianti F (2018) Activity of selected essential oils on spoiling fungi cultured from Marzolino cheese. Annals of Agricultural and Environmental Medicine 25, 280-284.

Papagianni M (2014) An evaluation of the proteolytic and lipolytic potential of Penicillium Spp. Isolated from traditional Greek sausages in submerged fermentation. Applied Biochemistry and Biotechnology 172, 767-775.

Pajonk AS, Saurel R and Andrieu J (2003) Experimental study and modeling of effective $\mathrm{NaCl}$ diffusion coefficients values during Emmental cheese brining. Journal of Food Engineering 60, 307-313.

Sacristán N, González L, Castro JM, Fresno JM and Tornadijo ME (2012) Technological characterization of Geotrichum candidum strains isolated from a traditional Spanish goats' milk cheese. Food Microbiology 30, $260-266$

Saurel R, Pajonk A and Andrieu J (2004) Modelling of French Emmental cheese water activity during salting and ripening periods. Journal of Food Engineering 63, 163-170.

Sousa MJ, Ardö Y and McSweeney PLH (2001) Advances in the study of proteolysis during cheese ripening. International Dairy Journal 11, 327-345.

Wolfe BE, Button JE, Santarelli $\mathbf{M}$ and Dhutton RJ (2014) Cheese rind communities provide tractable systems for in situ and in vitro studies of microbial diversity. Cell 158, 422-433. 